\title{
Prospección de lepidópteros antófagos asociados a Acacia macracantha Willd. (Fabaceae) en el norte de Chile
}

\author{
Héctor A. Vargas ${ }^{1} \&$ Luis E. Parra ${ }^{2}$
}

\begin{abstract}
${ }^{1}$ Departamento de Recursos Ambientales, Facultad de Ciencias Agronómicas, Universidad de Tarapacá, Casilla 6-D, Arica, Chile. havargas@uta.cl ${ }^{2}$ Departamento de Zoología, Facultad de Ciencias Naturales y Oceanográficas, Universidad de Concepción, Casilla 160-C, Concepción, Chile. luparra@udec.cl
\end{abstract}

\begin{abstract}
Survey of anthophagous lepidopteran larvae associated with Acacia macracantha Willd. (Fabaceae) in northern Chile. A survey of anthophagous lepidopteran larvae associated with Acacia macracantha Willd. (Fabaceae) was made in two coastal valleys from northern Chile: Azapa and Chaca. Eight species, distributed in eight genera and five families, were recorded in the areas. Species composition was the same for both localities. However, assemblage abundance and species abundance were different between the valleys.
\end{abstract}

KEYWORDS. Cosmopterigidae; Geometridae; Gracillariidae; Lycaenidae; Tortricidae.

RESUMEN. Prospección de lepidópteros antófagos asociados a Acacia macracantha Willd. (Fabaceae) en el norte de Chile. Se efectuó una prospección de larvas de lepidópteros antófagos asociados a Acacia macracantha Willd. (Fabaceae) en dos valles costeros del norte de Chile: Azapa y Chaca. Fueron registradas ocho especies, distribuidas en ocho géneros y cinco familias. La composición de especies del ensamble fue la misma en las dos localidades. Sin embargo, la abundancia del ensamble y la abundancia por especie fueron diferentes entre los valles.

PALABRAS CLAVE. Cosmopterigidae; Geometridae; Gracillariidae; Lycaenidae; Tortricidae.

El conocimiento sobre plantas hospederas de larvas de Lepidoptera de Chile es escaso. La mayoría de los registros publicados se refieren a especies con algún grado de importancia agrícola (Artigas 1994). Sin embargo, los registros de plantas hospederas de insectos fitófagos son importantes por diversos motivos (Monteiro et al. 2007). Un adecuado conocimiento de las relaciones insecto-planta permite estudiar la biología de las especies involucradas (Flinte et al. 2006), y de esta manera sirve como base para planificar la conservación biológica.

El yaro (Acacia macracantha Willd., Fabaceae) es una leguminosa arbórea que se distribuye ampliamente en América, por el norte tiene su límite en el sur de México y en el extremo sur de Florida, está presente en el Caribe y en las islas Galápagos, pero se encuentra especialmente representada a lo largo de la costa Pacífico de Sudamérica hasta el extremo norte de Chile (Aronson 1991). Además, existen registros desde Bolivia y Argentina (Cialdella 1984). En Chile su distribución geográfica natural está restringida a los valles costeros del extremo norte, donde forma parte de comunidades vegetales nativas (Aronson 1991). Sin embargo, en estos valles la mayor parte de la superficie cubierta originalmente por bosque espinoso tropical interior, donde se desarrolla A. macracantha, se encuentra actualmente sometida a uso agrícola (Luebert \& Pliscoff 2006). La pérdida de hábitat es una de las principales amenazas de extinción para insectos (Perlman \& Paskowitz 1998). Este es un factor que puede ser particularmente significativo en los valles costeros del norte de Chile, en la medida en que continúe la transformación del hábitat original.

La mayoría de las especies de Lepidoptera tienen larvas fitófagas. Esta es una de las razones de su importancia en ecosistemas naturales (Scoble 1995). Las especies con larvas antófagas son especialmente importantes en la biología reproductiva de las plantas como A. macracantha que se reproducen por semillas.

En este estudio se presentan los resultados de una prospección de lepidópteros antófagos asociados a inflorescencias de A. macracantha en los valles de Azapa y Chaca, Provincia de Arica, norte de Chile, con el objetivo de contribuir a caracterizar la composición de especies asociadas a estas inflorescencias, además de obtener una estimación preliminar de sus respectivos niveles de abundancia.

\section{MATERIALY MÉTODOS}

Los valles costeros del norte de Chile se encuentran bajo la influencia del bioclima tropical hiperdesértico (Leubert \& Pliscoff 2006). Azapa ( $18^{\circ} 34^{\prime} \mathrm{S}, 70^{\circ} 00^{\prime} \mathrm{O}$ ) presenta un alto grado de intervención antrópica derivada de la actividad agrícola; gran parte de su superficie se encuentra ocupada por diversos cultivos hortícolas y olivo (Olea europaea L., Oleaceae); la mayoría de los ejemplares de A. macracantha en esta localidad se presentan como plantas aisladas, ubicadas en orillas de caminos, bordes de campos agrícolas, lecho del río, o como ornamentales. Chaca $\left(18^{\circ} 48^{\prime} \mathrm{S}, 70^{\circ} 07^{\prime} \mathrm{O}\right)$ presenta un grado de intervención antrópica menor al del valle de Azapa, aunque igualmente existen algunos campos cultivados con hortalizas y olivos; sin embargo, A. macracantha se encuentra formando parte de parches con otras especies nativas, entre las que predominan especies arbustivas de la familia 
Asteraceae, especialmente Pluchea chingoyo D.C. Azapa y Chaca se encuentran separados por una distancia aproximada de 30 kilómetros de desierto.

Las colectas fueron efectuadas cada 15 días durante la época en que se concentra la floración principal de $A$. macracantha, entre diciembre-2002 y enero-2003. Cada localidad fue colectada tres veces. En cada oportunidad 10 árboles fueron seleccionados aleatoriamente por valle, totalizando 30 árboles muestreados por localidad. De cada árbol se tomaron al azar 50 inflorescencias a una altura aproximada de 1,50-1,90m. En laboratorio se cuantificó la abundancia larvaria de las especies presentes en cada muestra observando bajo lupa estereoscópica. Con la finalidad de confirmar la determinación taxonómica sobre la base de caracteres morfológicos imaginales, aproximadamente 200 larvas de las diferentes especies colectadas fueron mantenidas en crianza en laboratorio dentro de frascos de plástico, proporcionando periódicamente nuevas inflorescencias de $A$. macracantha como alimento, hasta que se obtuvieron pupas y posteriormente adultos. Los adultos emergidos fueron pinchados y etiquetados. Ejemplares de referencia quedaron depositados en la Colección Entomológica de la Universidad de Tarapacá (IDEA).

Con la finalidad de detectar posibles diferencias entre los valores de abundancia del ensamble y de abundancia por especie entre las localidades, se utilizó la prueba $U$ de MannWhitney $(\alpha=0,05)$. Mientras que para verificar si existió efecto de la localidad sobre la abundancia relativa de las especies del ensamble se aplicó una prueba de $\chi^{2}$ de independencia $(\alpha=$ $0,05)$. Los análisis estadísticos fueron efectuados con BioEstat 5.0 (Ayres et al. 2007).

\section{RESULTADOS YDISCUSIÓN}

Durante este estudio fueron examinadas 3.000 inflorescencias de A. macracantha. En ellas fueron colectadas 1.896 larvas antófagas de Lepidoptera: 1.308 en Chaca $(0,872$ larvas por inflorescencia) y 588 en Azapa (0,392 larvas por inflorescencia). Fueron identificadas ocho especies, distribuidas en ocho géneros y cinco familias.

Composición de especies. Las mismas especies fueron detectadas en ambas localidades: Ithome tiaynai Vargas, 2004 (Cosmopterigidae); Cyclophora nanaria (Walker, 1861) y Eupithecia yubitzae Vargas \& Parra, 2004 (Geometridae); Chileoptilia yaroella Vargas \& Landry, 2005 (Gracillariidae); Hemiargus ramon (Dognin, 1887), Leptotes trigemmatus (Butler, 1881) y Ministrymon azia (Hewitson, 1873) (Lycaenidae); y Cydia largo Heppner, 1981 (Tortricidae). Esta idéntica composición de especies puede ser entendida por la pertenencia de las localidades estudiadas a una misma unidad biogeográfica, tal como ha sido observado para otros taxa que se distribuyen a través del desierto costero del sur del Perú y norte de Chile (Porter, 1994; 1995a,b).

Abundancia. La abundancia de H. ramon y L. trigemmatus fue cuantificada para ambas especies en forma conjunta, dado que no fue posible separar las larvas de ellas bajo lupa

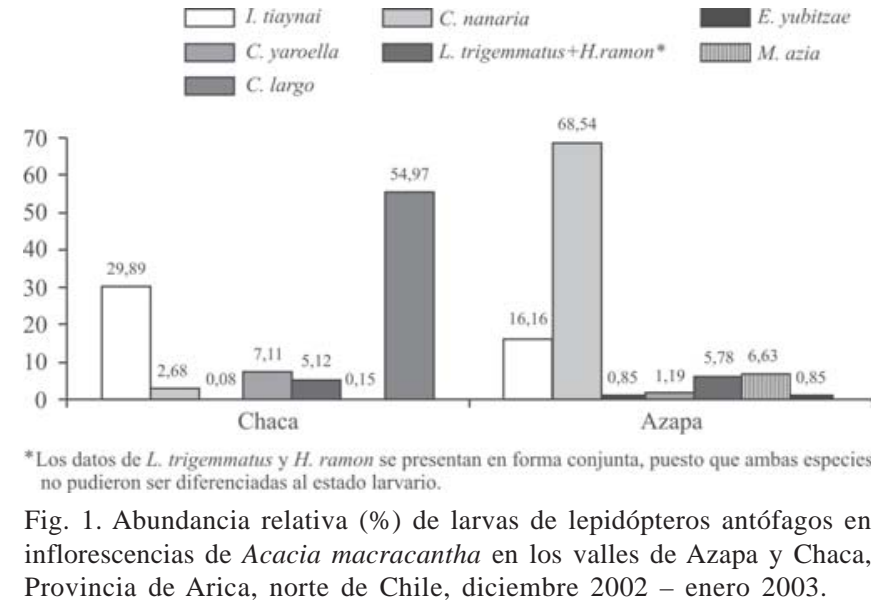

estereoscópica. El ensamble presentó mayor abundancia total en el valle de Chaca, $(U$-test $Z: 4,78014 ; P<0,0001$; Tabla I). Sin embargo, hubo una respuesta diferencial para la abundancia por especie en relación con la localidad (Tabla I): C. largo, I. tiaynai y $C$. yaroella fueron más abundantes en el valle de Chaca ( $U$-test. $Z$ : 6,090; $P<0,0001 ; Z: 5,2854 ; P<0,0001 ; Z$ : 4,$72 ; P<0,0001$, respectivamente, Tabla I), mientras que la abundancia de $C$. nanaria y $M$. azia fue mayor en el valle de Azapa ( $U$-test: $Z: 5,1154 ; \mathrm{P}<0,0001 ; Z: 2,0994 ; P=0,0179$, respectivamente, Tabla I). Por otro lado, la abundancia larvaria de E. yubitzae y L. trigemmatus $+H$. ramon no fue afectada por la localidad ( $U$-test: $Z$ : 0,2366 ; n.s; $Z: 1,3380$; n.s, respectivamente, Tabla I). En ambas localidades más del $80 \%$ de la abundancia fue explicada por sólo dos especies, mientras que la mayoría de las especies alcanzaron bajos niveles de abundancia dentro del ensamble. Al respecto, Barbosa et al. (2000) indicaron que la dominancia de ensambles de herbívoros por parte de relativamente pocas especies puede ser una característica común para varios hábitats.

Las tres especies que fueron más abundantes en Chaca (i.e.: C. largo, I. tiaynai y C. yaroella) tienen como único

Tabla I. Abundancia (media (desviación estándar)) de larvas de lepidópteros antófagos en 30 muestras de 50 inflorescencias de Acacia macracantha en los valles de Azapa y Chaca, Provincia de Arica, norte de Chile, durante diciembre 2002 - enero 2003.

\begin{tabular}{lcccc}
\hline ESPECIE & CHACA & AZAPA & $Z^{(2)}$ & $P^{(3)}$ \\
\hline I. tiaynai & $13,03(7,86)$ & $3,17(3,47)$ & 5,2854 & $<0,0001$ \\
C. nanaria & $1,17(1,42)$ & $13,43(13,22)$ & 5,1154 & $<0,0001$ \\
E. yubitzae & $0,03(0,18)$ & $0,17(0,65)$ & 0,2366 & n.s. \\
C. yaroella & $3,1(2,88)$ & $0,23(0,50)$ & 4,7236 & $<0,0001$ \\
L. trigemmatus $+^{2}$ & $2,23(3,37)$ & $1,13(1,61)$ & 1,3380 & n.s. \\
H. ramon & & & & \\
M. azia & $0,07(0,25)$ & $1,3(2,32)$ & 2,0994 & 0,0179 \\
C. largo $_{\text {TOTAL }}^{23,96(9,91)}$ & $0,17(0,59)$ & 6,4090 & $<0,0001$ \\
ENSAMBLE & $43,6(12,44)$ & $19,6(17,65)$ & 4,7014 & $<0,0001$
\end{tabular}

${ }^{1}$ Los datos de abundancia para L. trigemmatus y H. ramon se presentan juntos, pues ambas especies no pudieron ser separadas al estado larvario. ${ }^{2}$ Valores de $Z$ según Prueba $U$ de Mann-Whitney.

${ }^{3} \mathrm{Nivel}$ de significancia asociados al respectivo valor $Z$. 
hospedero en el área de estudio a A. macracantha (Vargas 2004, Vargas \& Landry 2005, Vargas \& Parra 2006). Esto sugiere que los niveles poblacionales de estas tres especies podrían verse disminuidos como una respuesta a los menores niveles poblacionales de su hospedero en Azapa. Sin embargo, el esclarecimiento de este patrón observado escapa al objetivo del presente trabajo. Por otro lado, las especies que presentaron mayor abundancia en Azapa y aquellas cuya abundancia no varió entre las dos localidades tienen adicionalmente otras plantas hospederas en el área de estudio.

Abundancia relativa. La abundancia relativa de las especies del ensamble fue afectada significativamente por la localidad $\left(\chi^{2}: 1.216,401 ;\right.$ g.1.: $\left.6 ; P<0,0001\right)$. En el valle de Chaca la mayor abundancia relativa fue para $C$. $\operatorname{largo}(54,97 \%$, Fig. 1), y la menor para E. yubitzae (0,08\%, Fig. 1). En el valle de Azapa la mayor abundancia relativa fue para $C$. nanaria $(68,54 \%$, Fig. 1), y la menor para C. largo y E. yubitzae (0,85\%, Fig. 1). Estos datos estarían reforzando la posibilidad de que las especies monófagas sean negativamente afectadas en un ambiente con menores niveles poblacionales del hospedero, tal como acontece en el valle de Azapa, donde la mayor abundancia relativa fue para $C$. nanaria, una especie polífaga en el área de estudio (Vargas et al. 2001). La abundancia relativa de las especies componentes de una comunidad puede ser modificada profundamente por distintos niveles de perturbación ambiental (Tovar-Sánchez et al. 2004), puesto que la densidad de una población es producto del grado de encaje entre su rango de tolerancia y el rango ambiental existente (Saiz 1980). Al respecto, Jiménez-Valverde et al. (2004) encontraron un efecto significativo de diferentes hábitats sobre la abundancia relativa de los componentes de comunidades de mariposas.

Los resultados presentados y discutidos en este trabajo abarcan una pequeña escala temporal. Sin embargo, ponen en evidencia la importancia que las inflorescencias de $A$. macracantha tienen como hospederas de lepidópteros antófagos silvestres en las localidades estudiadas, y constituyen las primeras estimaciones de abundancia de las especies antófagas asociadas a A. macracantha en el norte de Chile. Futuros estudios deberían emplear una escala temporal mayor, una detallada caracterización de la distribución y abundancia de otros hospederos de las especies del ensamble, y profundizar en el conocimiento sobre la biología de las diferentes especies detectadas.

Agradecimientos. A Bernard Landry, Muséum d'histoire naturelle, Ginebra, Suiza; Lázaro Roque-Albelo, Charles Darwin Research Station, Islas Galápagos, Ecuador; Amabílio J.A. de Camargo, Embrapa Cerrados, Planaltina, Brasil; Jonny E. Duque Luna y Jaime I. Rodríguez Fernández, Universidade Federal do Paraná, Curitiba, Brasil, por los comentarios y sugerencias efectuados sobre una versión preliminar. El apoyo financiero para esta investigación fue obtenido del Proyecto DIPOG 9704-03, de la Dirección de Investigación y Postgrado de la Universidad de Tarapacá, Arica, Chile. Los comentarios vertidos por dos revisores anónimos fueron especialmente útiles para generar la versión definitiva de este artículo.

\section{REFERENCIAS}

Aronson, J. 1991. Description and distribution of Acacia macracantha Humb. Et Bonpl. ex Willd. (Leguminosae, Mimosoideae) in northern Chile. Gayana Botánica 48: 81-88.

Artigas, J. N. 1994. Entomología Económica. Insectos de interés agrícola, forestal, médico y veterinario (nativos, introducidos o susceptibles de ser introducidos). Vol. II. Ediciones Universidad de Concepción. Concepción, Chile. 943 p.

Ayres, M.; M. Ayres Jr.; D. L. Ayres \& A. S. Santos. 2007. BioEstat 5.0 aplicações estatísticas nas áreas das ciências bio-médicas. Belém, Pará, Brasil.

Barbosa, P.; A. Zegarra \& P. Gross. 2000. Structure of two macrolepidopteran assemblages on Salix nigra (Marsh) and Acer negundo L.: abundance, diversity, richness, and persistence of scarce species. Ecological Entomology 25: 374-379.

Cialdella, A. M. 1984. El género Acacia (Leguminosae) en la Argentina. Darwiniana 21: 59-111.

Flinte, V.; C. O. Araujo; M. V. de Macedo \& R. F. Monteiro. 2006. Insetos fitófagos associados ao murici da praia, Byrsonima sericea (Malpighiaceae), na Restinga de Jurubatiba (RJ). Revista Brasileira de Entomologia 50: 512-523.

Jiménez-Valverde, A.; J. Martín-Cano \& M. L. Munguira. 2004. Patrones de diversidad de la fauna de mariposas del Parque Nacional de Cabañeros y su entorno (Ciudad Real, España central) (Lepidoptera, Papilionoidea, Hesperioidea). Animal Biodiversity and Conservation 27: 15-24.

Luebert, F. \& P. Pliscoff. 2006. Sinopsis bioclimática y vegetacional de Chile. Editorial Universitaria, Santiago, Chile. $316 \mathrm{p}$.

Monteiro, R. F.; M. V. Macedo; M. de S. Nascimento \& R. S. F. Cury. 2007. Composição, abundância e notas sobre a ecologia de espécies de larvas de lepidopteros associadas a cinco espécies de plantas hospedeiras no Parque Nacional da Restinga de Jurubatiba, R.J. Revista Brasileira de Entomologia 51: 476-483.

Perlman D. L. \& D. Paskowitz. 1998. Conservation and Biodiversity of Insects, p. 432-457. In: W. S. Romoser \& J. G. Stoffolano (ed.). The Science of Entomology, 4rth edition. Boston, Massachusetts, $605 \mathrm{p}$.

Porter, C. C. 1994. Perspectivas del control biológico en la Pampa del Tamarugal: aspectos biogeográficos y paleoecológicos. Idesia 23: $81-90$.

Porter, C. C. 1995a. A revision of Cosmiocryptus in the coastal desert of Perú and north Chile (Hymenoptera: Ichneumonidae). Psyche 92: 461-492.

Porter, C. C.1995b. Trachysphyrus and the new genus Aeliopotes in the costal desert of Perú and north Chile (Hymenoptera: Ichneumonidae). Psyche 92: 513-545.

Saíz, F. 1980. Experiencias en el uso de criterios de similitud en el estudio de comunidades. Archivos de Biología y Medicina Experimentales 13: 367-402.

Scoble, M. J. 1995. The Lepidoptera. Form, Function and Diversity. The Natural History Museum, London. Oxford University Press, Suffolk, London. 404 p.

Tovar-Sánchez, E.; Z. Cano-Santana \& K. Oyama. 2004. Canopy arthropod communities on Mexican oaks at sites with different disturbance regimes. Biological Conservation 115: 79-87.

Vargas, H. A. 2004. Una nueva especie de Ithome Chambers (Lepidoptera: Cosmopterigidae: Chrysopeleiinae) del norte de Chile. Revista Chilena de Historia Natural 77: 285-292.

Vargas, H. A.; H. E. Vargas; D. E. Bobadilla \& L. E. Parra. 2001. Notas sobre la polilla de la flor del olivo Cyclophora nanaria Walker (Lepidoptera: Geometridae: Sterrhinae). Idesia 19: 25-33.

Vargas, H. A. \& B. Landry. 2005. A new genus and species of Gracillariidae (Lepidoptera) feeding on flowers of Acacia macracantha Willd. (Mimosaceae) in Chile. Acta Entomológica Chilena 29: 47-57.

Vargas, H. A. \& L. E. Parra. 2006. Nuevos registros de distribución y notas biológicas de Cydia largo Heppner (Lepidoptera: Tortricidae). Gayana 70: 293-294. 